\title{
Potência Elétrica Irradiada e Potência Mecânica Dissipada no Sistema de Dois Dipolos Oscilantes Próximos e em Fase
}

Irradiated and dissipated mechanical power of a system of two nearby parallel oscillating dipoles moving in phase

\author{
G. F. Leal Ferreira \\ Instituto de Física de São Carlos, USP, CP 369, 13560-970, São Carlos, SP \\ guilherm@if.sc.usp.br
}

Recebido em 08 de março, 2003. Aceito em 25 de julho, 2003.

\begin{abstract}
Mostra-se diretamente que a potência média irradiada por um sistema de dois dipolos oscilantes próximos, paralelos e em fase, é igual à potência mecânica média dissipada nos osciladores necessária para manter 0 movimento harmônico simples.

We show directly that the mean irradiated power from a system of two nearby parallel oscillators in phase is equal to the mean mechanical power supplied to both oscillators to mantain their harmonic motion.
\end{abstract}

\section{Introdução}

Em interessante artigo publicado nesta revista, o problema do acoplamento entre fontes radiantes foi abordado [1]. O autor chama a atenção para o fato de que a variação da potência total irradiada por um sistema de fontes em relação àquela como se fossem isoladas se deve à interação entre elas. Ocorreu-nos a idéia de comprovar diretamente a assertiva com um sistema físico simples, o de dois dipolos próximos, paralelos, com movimento harmônico simples (mhs) em fase. O ponto realmente sutil é que a energia irradiada depende do fluxo do vetor de Poynting dos campos longínquos em esfera distante, enquanto que a interação entre as fontes depende dos campos próximos ${ }^{2}$. Vai-se mostrar por cálculo direto que a potência total média irradiada pelos dois dipolos é igual à potência mecânica média empregada para promover a emissão como se fossem isoladas, adicionada da potência mecânica necessária média para cada oscilador executar o seu movimento em presença do outro. Ver-se-á que com os dipolos próximos, a potência emitida é quase quatro vezes a potência de emissão como isoladas, contrariando a visão de que, '.....como és claro, la energia total emitida por las dos fuentes és solo el doble de la que emite una solo fuente.' [2]. O cálculo será realizado para dipolos 'próximos', que significará aqui em segunda ordem da razão distância/ comprimento de onda.

\section{O campo elétrico do dipolo radi- ante}

A situação a ser estudada é a da emissão de um par de dipolos oscilantes, mhs, paralelos, de momento $M_{0}$, frequência angular $\omega$, como mostra a Fig. 1. Por razão da especial simetria estudada, só necessitaremos da componente $E_{\vartheta}$ do campo elétrico do dipolo [3], Fig.2,

$$
E_{\vartheta}=M_{0} \beta^{3} \operatorname{sen} \vartheta e^{i(\omega t-\beta r)}\left[\frac{-1}{\beta r}+\frac{i}{(\beta r)^{2}}+\frac{1}{(\beta r)^{3}}\right]
$$

$\operatorname{com} \beta=\omega / c=2 \pi / \lambda$, sendo $\lambda$ o comprimento de onda da radiação.

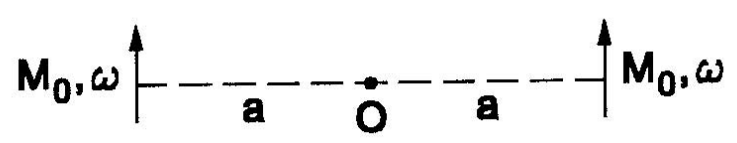

Figura 1. Dois dipolos oscilantes, paralelos, de momento $M_{0}$, em mhs com frequência $\omega$, distantes $a$ do ponto médio $\mathrm{O}$.

\section{A potência irradiada pelo sistema de dois dipolos}

Para o estudo da potência irradiada pelos dipolos da Fig. 1, consideremos a Fig. 3. O campo elétrico total em pontos distantes será a soma dos campos de cada um dos dipolos, tendo em conta a diferença de fase devido à diferença de distância, $\Delta r$, entre os dipolos e o ponto considerado. Em pontos distantes tomam-se os campos com a dependência em $1 / r$. Tendo como referência o ponto médio $O$ entre as fontes, o campo elétrico total $E_{\vartheta T}$ na direção $\vartheta$ em $r_{0}$ será

$$
E_{\vartheta T}=-\frac{M_{0} \beta^{2} \operatorname{sen} \vartheta e^{i\left(\omega t-\beta r_{0}\right)}}{r}\left(e^{i \beta \Delta r}-e^{-i \beta \Delta r}\right)
$$




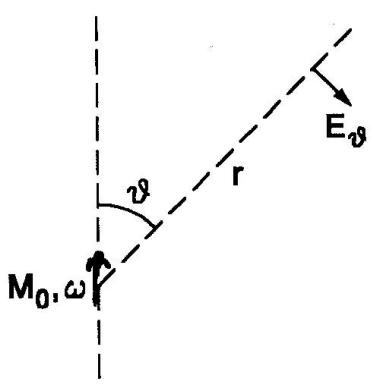

Figura 2. Componente $\vartheta$ do campo elétrico do dipolo oscilante.

com

$$
\Delta r=a \operatorname{sen} \vartheta,
$$

sendo $2 a$ a distância entre as fontes. $\mathrm{Na}$ aproximação $\beta a<<1$, a Eq. 2 é

$$
E_{\vartheta T}=-\frac{M_{0} \beta^{2} \operatorname{sen} \vartheta e^{i\left(\omega t-\beta r_{0}\right)}}{r}\left(2-\beta^{2} \Delta r^{2}\right)
$$

e o fluxo do vetor de Poynting médio, $P$, numa esfera de raio $\rightarrow \infty$ será

$$
P=\frac{c}{2} \operatorname{Re} \int_{0}^{\pi} \frac{E_{\vartheta T} E_{\vartheta T}^{*} r^{2} \operatorname{sen} \vartheta d \vartheta}{4 \pi}
$$

ou pela Eq. 4,

$$
P=\frac{M_{0}^{2} \beta^{4} c}{8 \pi} \int_{0}^{\pi}\left(4-4 \beta^{2} a^{2} \operatorname{sen}^{2} \vartheta\right) \operatorname{sen}^{3} \vartheta d \vartheta
$$

que dá finalmente

$$
P=\frac{M_{0}^{2} \beta^{3} \omega}{2 \pi}\left(\frac{4}{3}-\frac{8}{15} \beta^{2} a^{2}\right)
$$

tendo em conta que $\beta=\omega / c$. Notemos que a potência média irradiada por um dipolo isolado, $P_{1}$, pode ser obtido da Eq. 7 acima, fazendo-se $a=0$ e dividindo-se o resultado por 4 já que $P$ se refere à emissão de dois dipolos e é proporcional ao quadrado de $M_{0}$, ou seja,

$$
P_{1}=\frac{M_{0}^{2} \beta^{3} \omega}{6 \pi}
$$

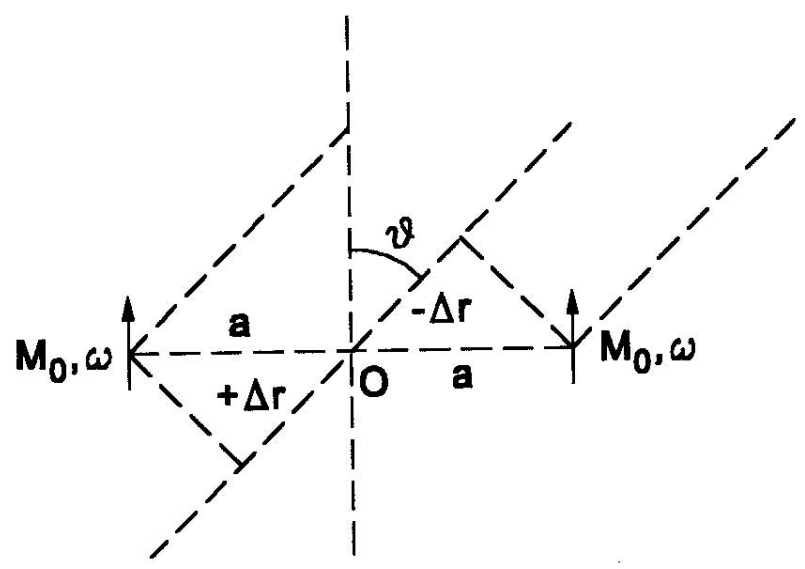

Figura 3. Diferença de percurso, $\pm \Delta r= \pm a$ sen $\vartheta$, contado a partir de $\mathrm{O}$, para o cálculo da interferência dos campos de radiação dos dois dipolos.

Para $a$ pequeno, $P$ é quase quatro vezes maior que $P_{1}$ (e não duas, como mencionado em [2]), mas como enfatizado em [1], isto se deve a uma maior potência mecânica empregada para manter os osciladores em mhs, como veremos.

\section{Ação de um dipolo sobre o outro}

Consideremos agora o campo elétrico $E_{e d}$ que o dipolo da esquerda da Fig. 1 cria sobre o dipolo da direita. Tomando o sentido positivo para cima, ele é $-E_{\vartheta}\left(r=2 a, \vartheta=90^{\circ}\right)$, com $E_{\vartheta}$ tirado da Eq. 1. O trabalho por ciclo, $W$, executado por esta força será calculado de $q \int E_{\text {ed }} v d t$ num período, sendo $q$ a carga do dipolo e $v$ a velocidade, igual a $i \omega M_{0} e^{i \omega t} / q$, que em AC é dado por

$$
W=\frac{-1}{2} \operatorname{Re} E_{e d} \cdot v^{*}=\frac{-1}{2} M_{0}^{2} \beta^{3} \omega \operatorname{Re}\left[(\cos 2 \beta a-i \operatorname{sen} 2 \beta a)\left(\frac{-1}{\beta r}+\frac{i}{(2 \beta a)^{2}}+\frac{1}{(2 \beta a)^{3}}\right) i\right]
$$

Fazendo $Y=\operatorname{Re} E_{e d} v^{*}$, temos

$$
Y=-(\operatorname{sen} 2 \beta a)\left(\frac{-1}{2 \beta a}+\frac{1}{(2 \beta a)^{3}}\right)-\frac{\cos 2 \beta a}{(2 \beta a)^{2}}
$$

Para obtermos $Y$ correto até termos de $2^{a}$ ordem em $\beta a$, sendo $\beta a<<1$, devemos usar para $\operatorname{sen} x$ até termos de $5^{a}$ ordem, ou seja $x-x^{3} / 3$ ! $+x^{5} / 5$ ! e para o $\cos x$, termos até $4^{a}$, ou seja, $1-x^{2} / 2+x^{4} / 4$ !. Feito isto, obtém-se

$$
Y=\frac{2}{3}-\frac{8 \beta^{2} a^{2}}{15}
$$

e $W$ da Eq. 8 será

$$
W=-M_{0}^{2} \beta^{3}\left(\frac{1}{3}-\frac{4 a^{2} \beta^{2}}{15}\right) .
$$

A potência média será $P^{\prime}=W / T=W \omega / 2 \pi$, sendo $T$ o período de oscilação. Temos então

$$
P^{\prime}=-M_{0}^{2} \beta^{3} \frac{\omega}{2 \pi}\left(\frac{1}{3}-\frac{4 a^{2} \beta^{2}}{15}\right) .
$$

O sinal negativo em $P^{\prime}$ significa que o agente mecânico agindo sobre o oscilador à direita deve exercer trabalho extra para mantê-lo em mhs. 


\section{Potência irradiada e potência mecânica nos osciladores}

$P^{\prime}$ na Eq. 12 dá a potência média que deve ser exercida sobre o oscilador da direita (esquerda), sob a ação do da esquerda (direita) para que mantenha o mhs. Queremos calcular a potência mecânica média total agindo sobre cada oscilador, $P_{m}$, para compararmos com $P$ da Eq. 7. Para isto deveremos adicionar à $P^{\prime}$ a potência necessária para ele emitir como se fosse isolado, que está dado na Eq. 8, com $P_{1}$. Pode-se objetar que este resultado foi obtido com o auxílio dos campos em pontos distantes, Eq. 2, e é na verdade potência emitida e não potência mecânica dissipada. Mas note-se que a Eq. 12, que se refere à potência dissipada, fornece o mesmo resultado fazendo-se $a=0$. Isto é, com $a=0$, os dipolos coalescem e a interação mútua torna-se auto-interação. Teremos então para $P_{m}$

$$
P_{m}=P^{\prime}+P_{1}=M_{0}^{2} \beta^{3} \frac{\omega}{2 \pi}\left(\frac{2}{3}-\frac{4 a^{2} \beta^{2}}{15}\right) .
$$

A potência mecânica média total, $P_{m T}$, será $2 P_{m}$, ou seja,

$$
P_{m T}=M_{0}^{2} \beta^{3} \frac{\omega}{2 \pi}\left(\frac{4}{3}-\frac{8 a^{2} \beta^{2}}{15}\right)
$$

que é igual a $P$, potência irradiada pelo sistema, Eq. 7.

\section{Considerações finais}

Demonstrou-se diretamente que a potência irradiada pelo sistema de dois dipolos em fase vem da potência mecânica média empregada em cada um deles para emitir mantendo o mhs, sobrecarregada pela ação do dipolo companheiro. O cálculo mostra a consistência interna da Teoria do Eletromagnetismo, visto que diferentes expressões do campo elétrico são empregadas. Seria interessante estendê-lo além do termo em $(\beta a)^{2}$.

\section{Referências}

1. Reinaldo Welti, Rev. Bras. Ens. Física, 24, 415 (2002).

2. Citado em [1].

3. J. C. Slater, Microwave Transmission, Dover Public., 1942, Cap.V. 